\title{
Listeriosis outbreak caused by acid curd cheese 'Quargel', Austria and Germany 2009
}

R Fretz (rainer.fretz@ages.at) ${ }^{1}$, U Sagel ${ }^{1,2}$, W Ruppitsch ${ }^{1}$, A T Pietzka ${ }^{1}$, A Stöger ${ }^{1}$, S Huhulescu ${ }^{1}$, S Heuberger ${ }^{1}$, J Pichler ${ }^{1}$, P Much ${ }^{1}$, G Pfaff ${ }^{3}$, K Stark $^{4}$, R Prager ${ }^{4}$, A Flieger ${ }^{4}$, 0 Feenstra ${ }^{5}$, F Allerberger ${ }^{1}$

1. Austrian Agency for Health and Food Safety (AGES), Vienna, Austria

2. Binational Consiliar Laboratory for Listeria, Germany and Austria, Vienna, Austria

3. State Health Office (LGA) Baden-Württemberg, Stuttgart, Germany

4. Robert Koch Institute (RKI), Berlin and Wernigerode, Germany

5. Public Health Authority, Graz, Austria

Citation style for this article:

Citation style for this article: Fretz R, Sagel U, Ruppitsch W, Pietzka AT, Stöger A, Huhulescu S, Heuberger S, Pichler J, Much P, Pfaff G, Stark K, Prager R, Flieger A, Feenstra O, Allerberger F. Listeriosis outbreak caused by acid curd cheese 'Quargel', Austria and Germany 2009. Euro Surveill. 2010;15(5):pii=19477. Available online: http://www.eurosurveillance.org/ViewArticle.aspx?Articleld=19477

We report an outbreak of listeriosis in Austria and Germany due to the consumption of 'Quargel' cheese produced by an Austrian manufacturer. At the time of writing this report, the outbreak was known to account for 14 outbreak cases in 2009 , including four cases with lethal outcome. On 23 January 2010, the cheese product was voluntarily withdrawn from the market.

On 14 August 2009, the binational Austrian-German Consiliar Laboratory for Listeria in Vienna noticed the occurrence of a new pulsed-field gel electrophoresis (PFGE) pattern in human isolates of Listeria monocytogenes serotype $1 / 2 a$. This consiliar laboratory receives all human isolates from Austria as required by law. In Germany, submission of isolates is voluntary. According to the available information at the time of writing this report, the outbreak clone accounted for 12 of the 46 Austrian cases in 2009 (serotype $1 / 2 a(n=29), 4 b(n=9), 1 / 2 b(n=8))$. Onset of illness is shown in the Figure. The 12 Austrian outbreak cases (two of them fatal) affected six of nine Austrian provinces. The mean age was 74.5 years (range: $58-88$ years), eleven patients were male. In addition, two of 92 available human isolates from Germany in 2009 (total number of cases 389) showed this new PFGEpattern. The German outbreak cases were two women in their 70s who died in November and December 2009 respectively. They had not visited Austria during the likely period of incubation (up to 70 days).

Since no reliable information was available on food consumed during the incubation period, all surviving Austrian outbreak cases were asked to collect grocery receipts for the three weeks after 3 December, i.e. after they were discharged from hospital, in order to collect information on routine food consumption behaviour. This epidemiological investigation revealed consumption of 'Quargel', a type of acid curd cheese available in different flavours, as a highly likely source of this outbreak. Three of seven outbreak cases providing receipts had bought product $X$ produced by the Austrian manufacturer. Regular consumption of Quargel product X was confirmed by eight of nine participating outbreak cases, and consumption of Quargel cheese products was reported by heteroanamnesis for one German outbreak case (data on the second case remain unavailable).

Approximately 16 tons of Quargel per week are produced by the Austrian manufacturer. Fifty-three per cent of the product is exported to the German market and small amounts to the Czech Republic, Poland and Slovakia. This cheese is made of curdled milk, which ripens after addition of starter cultures for one day at $28^{\circ} \mathrm{C}$, and after being sprayed with Brevibacterium linens for another two days at $14^{\circ} \mathrm{C}$. The shelf life after packing and marketing is two months.

An environmental L. monocytogenes $1 / 2 \mathrm{a}$ isolate from the production plant, collected in December 2009, became available in January 2010 and proved indistinguishable from the outbreak strain by genotyping. Quargel cheese products sampled at the plant on January 13 yielded three different strains of

\section{FIGURE}

Outbreak cases of listeriosis by onset of illness, Austria and Germany, $2009(\mathrm{n}=14)$




L. monocytogenes $1 / 2 \mathrm{a}$, including the outbreak clone, in numbers of less than 100 colony-forming units (cfu) per gram. Food products collected on 18 January 2010 yielded greater than $100 \mathrm{cfu} / \mathrm{g} \mathrm{L}$. monocytogenes. The product was voluntarily withdrawn from the market on 23 January. On the same day, the public was informed about the incident and warned about cheese already bought. The plant stopped production. Investigation of the source of contamination is ongoing.

\section{Conclusion}

Industrial food production combined with international marketing of food and the low attack rate of L. monocytogenes hinder epidemiological outbreak investigation with traditional concepts [1]. Genotyping of $L$. monocytogenes isolates from clinical specimens can discriminate single-source clusters of food-borne infection and contribute to the identification and investigation of outbreaks. The outbreak described in this report probably would not have been identified without molecular typing [2]. The effectiveness of microbiological surveillance is entirely dependent upon the consistent and timely submission of all Listeria isolates from clinical laboratories to public health laboratories. In Austria, clinical laboratories are required by law to submit all clinical isolates of $L$. monocytogenes to AGES for PFGE analysis. In Germany, submission of L. monocytogenes isolates from clinical specimens by clinical laboratories is not required. The high case fatality ratio of listeriosis makes a strong case for the importance and priority of improved surveillance in Europe [3]. Our outbreak report underlines the value of routine molecular typing of Listeria isolates and also points out the considerable potential of cross-border cooperation for elucidating chains of infections.

\section{References}

1. Allerberger F, Wagner M. Listeriosis: a resurgent foodborne infection. Clin Microbiol Infect. 2010;16(1):16-23.

2. Centers for Disease Control and Prevention (CDC). Outbreak of Listeria monocytogenes Infections Associated With Pasteurized Milk From a Local Dairy-Massachusetts, 2007. MMWR Morb Mortal Wkly Rep. 2008;57(40):1097-100.

3. Denny J, McLauchlin J. Human Listeria monocytogenes infections in Europe - an opportunity for improved European surveillance. Euro Surveill. 2008;13(13):pii=8082. Available from: http://www.eurosurveillance.org/ViewArticle. aspx?Articleld $=8082$ 